\title{
Lower-Inner Quadrant of the Breast
}

National Cancer Institute

\section{Source}

National Cancer Institute. Lower-Inner Quadrant of the Breast. NCI Thesaurus. Code C12302.

The quarter of the breast which is inferior and medial. 